\title{
On Zeta Regularization and Some of its Uses in Cosmology
}

\author{
Emilio Elizalde ${ }^{* \dagger}$ \\ Consejo Superior de Investigaciones Científicas \\ Instituto de Ciencias del Espacio \\ Institut d'Estudis Espacials de Catalunya (IEEC/CSIC) \\ Campus UAB, Facultat de Ciències, Torre C5-Parell-2a planta \\ 08193 Bellaterra (Barcelona) Spain \\ E-mail: elizaldedieec.uab.es
}

\begin{abstract}
Zeta regularization has been proven to be a fine, powerful and very reliable tool for the regularization of the vacuum energy density in ideal situations. With the additional help of the Hadamard calculus, we have shown it to yield also finite and physically meaningful answers in more involved cases, as when imposing physical boundary conditions in two- and higher-dimensional surfaces, being then able to mimic in a convenient way other ad hoc cut-offs, as non-zero depths. These recent developments are described in the first part of this presentation. Recently, those techniques have also been used in calculations of the contribution of the vacuum energy of the quantum fields which are presumably pervading the universe, to the cosmological constant. Naive calculations of the absolute contributions of all known fields lead to a value which is off by roughly 120 orders of magnitude, as compared with the results obtained from observational fits, what is known as the new cosmological constant problem. This is very difficult to solve and we address here such issue only indirectly, by means of some specific examples.
\end{abstract}

Fifth International Conference on Mathematical Methods in Physics

24-28, April 2006

Rio de Janeiro, Brazil

\footnotetext{
* Speaker.

${ }^{\dagger}$ Presently on leave at Dipartimento di Fisica, Università di Trento, Via Sommarive 14, I-38050 Povo (Italy).
} 


\section{Introduction}

This material corresponds to my lecture, on some basic mathematics which are not sufficiently well known in general to the theoretical physicist, and are central in order to deal, in a precise way, with modern approaches to some outstanding processes in Quantum Field Physics and Cosmology. All those have in common the fundamental use which is done of quantum fluctuations in the physical vacuum of the quantum field theory under consideration, be at a nano-electro-mechanical (NEM) or at a cosmological level. This is, to start with, the beauty of such mathematical methods, that has been indeed recognized by many authors, in particular, and at the very beginning, by Stephen Hawking in a seminal paper he wrote some thirty years ago [1].

We start by reviewing the definition of the concept of a pseudodifferential operator (YDO) and of the zeta function $\zeta_{A}(s)$ associated to a $\Psi D O A$. We continue with the definitions of the determinant and trace of $A$, obtained in a unique way from the concept of Wodzicki residue. After considering in more detail the singularity structure of the zeta function $\zeta_{A}(s)$, we address the issue of the multiplicative (or noncommutative) anomaly (or defect) of the determinant. We then change subject by considering a special but very important case in the zeta function family analysis, that is, the celebrated Chowla-Selberg expansion formula (CS) and the non-trivial generalizations thereof obtained by the author. At the last part of the paper, we addressed specific physical applications of these mathematics to the calculation of what has become to be called the cosmo-topological Casimir effect, that is the possible influence of the fluctuations of the quantum vacuum of some (scalar) fields at cosmological level, coming from the non-trivial topology of our spacetime. This is a rather old issue [2], but treated here under new eyes that connect with very recent approaches to the problem [3]. These ideas could be at the very origin of the acceleration in the expansion of the universe that has been observed recently and could, at least in part, be an ingredient of the so-called dark energy component that pervades the universe we are living in.

\section{YDOs, zeta functions, determinants, and traces}

A pseudodifferential operator $A$ of order $m$ on a manifold $M_{n}$ is defined through its symbol $a(x, \xi)$, which is a function belonging to the space $S^{m}\left(\mathbb{R}^{n} \times \mathbb{R}^{n}\right)$ of $\mathbb{C}^{\infty}$ functions such that for any pair of multi-indexs $\alpha, \beta$ there exists a constant $C_{\alpha, \beta}$ so that $\left|\partial_{\xi}^{\alpha} \partial_{x}^{\beta} a(x, \xi)\right| \leq C_{\alpha, \beta}(1+|\xi|)^{m-|\alpha|}$. The definition of $A$ is given, in the distribution sense, by

$$
A f(x)=(2 \pi)^{-n} \int e^{i<x, \xi>} a(x, \xi) \hat{f}(\xi) d \xi,
$$

$f$ is a smooth function, $f \in \mathscr{S}$; remember $\mathscr{S}=\left\{f \in C^{\infty}\left(\mathbb{R}^{n}\right) ; \sup _{x}\left|x^{\beta} \partial^{\alpha} f(x)\right|<\infty, \forall \alpha, \beta \in \mathbb{R}^{n}\right\}$, $\mathscr{S}^{\prime}$ being the space of tempered distributions and $\hat{f}$ the Fourier transform of $f$. When $a(x, \xi)$ is a polynomial in $\xi$ one gets a differential operator. In general, the order $m$ can be complex. The symbol of a $\Psi$ DO has the form $a(x, \xi)=a_{m}(x, \xi)+a_{m-1}(x, \xi)+\cdots+a_{m-j}(x, \xi)+\cdots$, being $a_{k}(x, \xi)=b_{k}(x) \xi^{k} . a(x, \xi)$ is said to be elliptic if it is invertible for large $|\xi|$ and if there exists a constant $C$ such that $\left|a(x, \xi)^{-1}\right| \leq C(1+|\xi|)^{-m}$, for $|\xi| \geq C$. An elliptic $\Psi D O$ is one with an elliptic symbol.

Pseudodifferential operators are useful tools, both in mathematics and in physics. They were crucial for the proof of the uniqueness of the Cauchy problem [4] and also for the proof of the 
Atiyah-Singer index formula [5]. In quantum field theory they appear in any analytical continuation process (as complex powers of differential operators, like the Laplacian) [6]. And they constitute nowadays the basic starting point of any rigorous formulation of quantum field theory through microlocalization, a concept that is considered to be the most important step towards the understanding of linear partial differential equations since the invention of distributions [7].

\subsection{The zeta function}

Let $A$ a positive-definite elliptic $\Psi D O$ of positive order $m \in \mathbb{R}$, acting on the space of smooth sections of $E$, an $n$-dimensional vector bundle over $M$, a closed $n$-dimensional manifold. The zeta function $\zeta_{A}$ is defined as

$$
\zeta_{A}(s)=\operatorname{tr} A^{-s}=\sum_{j} \lambda_{j}^{-s}, \quad \operatorname{Re} s>\frac{n}{m} \equiv s_{0} .
$$

where $s_{0}=\operatorname{dim} M /$ ord $A$ is called the abscissa of convergence of $\zeta_{A}(s)$. Under these conditions, it can be proven that $\zeta_{A}(s)$ has a meromorphic continuation to the whole complex plane $\mathbb{C}$ (regular at $s=0$ ), provided that the principal symbol of $A$ (that is $a_{m}(x, \xi)$ ) admits a spectral cut: $L_{\theta}=$ $\left\{\lambda \in \mathbb{C} ; \operatorname{Arg} \lambda=\theta, \theta_{1}<\theta<\theta_{2}\right\}, \operatorname{Spec} A \cap L_{\theta}=\emptyset$ (Agmon-Nirenberg condition). The definition of $\zeta_{A}(s)$ depends on the position of the cut $L_{\theta}$. The only possible singularities of $\zeta_{A}(s)$ are simple poles at $s_{k}=(n-k) / m, k=0,1,2, \ldots, n-1, n+1, \ldots$ M. Kontsevich and S. Vishik have managed to extend this definition to the case when $m \in \mathbb{C}$ (no spectral cut exists) [8].

\subsection{The zeta determinant}

Let $A$ a $\Psi D O$ operator with a spectral decomposition: $\left\{\varphi_{i}, \lambda_{i}\right\}_{i \in I}$, where $I$ is some set of indices. The definition of determinant starts by trying to make sense of the product $\prod_{i \in I} \lambda_{i}$, which can be easily transformed into a 'sum': $\ln \prod_{i \in I} \lambda_{i}=\sum_{i \in I} \ln \lambda_{i}$. From the definition of the zeta function of $A: \zeta_{A}(s)=\sum_{i \in I} \lambda_{i}^{-s}$, by taking the derivative at $s=0: \zeta_{A}^{\prime}(0)=-\sum_{i \in I} \ln \lambda_{i}$, we arrive to the following definition of determinant of $A$ [9]:

$$
\operatorname{det}_{\zeta} A=\exp \left[-\zeta_{A}^{\prime}(0)\right]
$$

An older definition (due to Weierstrass) is obtained by subtracting in the series above (when it is such) the leading behavior of $\lambda_{i}$ as a function of $i$, as $i \rightarrow \infty$, until the series $\sum_{i \in I} \ln \lambda_{i}$ is made to converge. The shortcoming is here — for physical applications — that these additional terms turn out to be non-local and, thus, are non-admissible in any renormalization procedure [10].

In algebraic QFT, in order to write down an action in operator language one needs a functional that replaces integration. For the Yang-Mills theory this is the Dixmier trace, which is the unique extension of the usual trace to the ideal $\mathscr{L}^{(1, \infty)}$ of the compact operators $T$ such that the partial sums of its spectrum diverge logarithmically as the number of terms in the sum: $\sigma_{N}(T) \equiv \sum_{j=0}^{N-1} \mu_{j}=$ $\mathscr{O}(\log N), \mu_{0} \geq \mu_{1} \geq \cdots$ The definition of the Dixmier trace of $T$ is: $\operatorname{Dtr} T=\lim _{N \rightarrow \infty} \frac{1}{\log N} \sigma_{N}(T)$, provided that the Cesaro means $M(\sigma)(N)$ of the sequence in $N$ are convergent as $N \rightarrow \infty$ (remember that: $\left.M(f)(\lambda)=\frac{1}{\ln \lambda} \int_{1}^{\lambda} f(u) \frac{d u}{u}\right)$. Then, the Hardy-Littlewood theorem can be stated in a way that connects the Dixmier trace with the residue of the zeta function of the operator $T^{-1}$ at $s=1$ (see Connes [11]): $\operatorname{Dtr} T=\lim _{s \rightarrow 1^{+}}(s-1) \zeta_{T^{-1}}(s)$. 


\subsection{The Wodzicki residue}

The Wodzicki (or noncommutative) residue [12] is the only extension of the Dixmier trace to

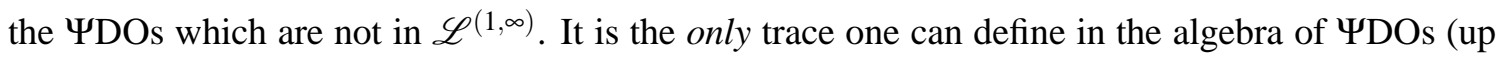
to a multiplicative constant), its definition being: res $A=2 \operatorname{Res}_{s=0} \operatorname{tr}\left(A \Delta^{-S}\right)$, with $\Delta$ the Laplacian. It satisfies the trace condition: res $(A B)=$ res $(B A)$. A very important property is that it can be expressed as an integral (local form) res $A=\int_{S^{*} M}$ tr $a_{-n}(x, \xi) d \xi$ with $S^{*} M \subset T^{*} M$ the co-sphere bundle on $M$ (some authors put a coefficient in front of the integral: Adler-Manin residue).

If $\operatorname{dim} M=n=-\operatorname{ord} A$ ( $M$ compact Riemann, $A$ elliptic, $n \in \mathbb{N}$ ) it coincides with the Dixmier trace, and one has $\operatorname{Res}_{s=1} \zeta_{A}(s)=\frac{1}{n}$ res $A^{-1}$. The Wodzicki residue continues to make sense for

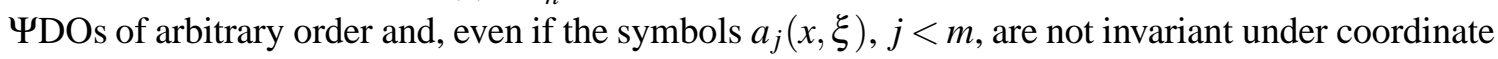
choice, their integral is, and defines a trace. All residua at poles of the zeta function of a $\Psi D O$ can be easily obtained from the Wodzciki residue [13].

\subsection{The multiplicative anomaly and its implications}

Given $A, B$ and $A B \Psi D O s$, even if $\zeta_{A}, \zeta_{B}$ and $\zeta_{A B}$ exist, it turns out that, in general, $\operatorname{det}_{\zeta}(A B) \neq$ $\operatorname{det}_{\zeta} A \operatorname{det}_{\zeta} B$. The multiplicative (or noncommutative) anomaly (or defect) is defined as:

$$
\delta(A, B)=\ln \left[\frac{\operatorname{det}_{\zeta}(A B)}{\operatorname{det}_{\zeta} A \operatorname{det}_{\zeta} B}\right]=-\zeta_{A B}^{\prime}(0)+\zeta_{A}^{\prime}(0)+\zeta_{B}^{\prime}(0)
$$

Wodzicki's formula for the multiplicative anomaly $[12,14,15]$ :

$$
\delta(A, B)=\frac{\operatorname{res}\left\{[\ln \sigma(A, B)]^{2}\right\}}{2 \operatorname{ord} A \operatorname{ord} B(\operatorname{ord} A+\operatorname{ord} B)}, \quad \sigma(A, B):=A^{\operatorname{ord} B} B^{-\operatorname{ord} A} .
$$

At the level of Quantum Mechanics (QM), where it was originally introduced by Feynman, the path-integral approach is just an alternative formulation of the theory. In QFT it is much more than this, being in many ocassions the actual formulation of QFT [16]. In short, consider the Gaussian functional integration $\int[d \Phi] \exp \left\{-\int d^{D} x\left[\Phi^{\dagger}(x)(\quad) \Phi(x)+\cdots\right]\right\} \longrightarrow \operatorname{det}()^{ \pm}$, and assume that the operator matrix has the following structure (being each $A_{i}$ an operator): $\left(\begin{array}{ll}A_{1} A_{2} \\ A_{3} & A_{4}\end{array}\right) \longrightarrow$ $\left(\begin{array}{ll}A & \\ & B\end{array}\right)$, where the last expression is the result of diagonalizing the operator matrix. A question now arises. What is the determinant of the operator matrix: $\operatorname{det}(A B) \operatorname{or} \operatorname{det} A \cdot \operatorname{det} B ?$ This has been very much on discussion $[17,18])$.

\subsection{A word on determinants}

Many fundamental calculations of QFT reduce, in essence, to the computation of the determinant of some suitable operator: at one-loop order, any such theory reduces in fact to a theory of determinants. The operators involved are pseudodifferential ( $\Psi D O)$, in loose terms 'some analytic functions of differential operators' (such as $\sqrt{1+D}$ or $\log (1+D)$, but $n o t \log D$ ). This is explained in detail in [19]. It is surprising that this seems not to be a main subject of study among mathematicians. I am referring to the determinants that involve in its definition some kind of regularization 
(related to operators that are not trace-class). This piece of calculus falls outside the scope of the standard disciplines and even many physically oriented mathematicians know little about this. The subject has many things in common with divergent series but lacks any reference comparable to the book of Hardy [20]. Actually, this question was already addressed by Weierstrass in a way not without problems, since it leads to non-local contributions that cannot be given a physical meaning in QFT. For completion, let us mention the well established theories of determinants for degenerate operators, for trace-class operators in the Hilbert space, Fredholm operators, etc. [21]

\subsection{On the method of zeta-function regularization}

Hawkwing introduced zeta-function regularization [1] to deal with infinities in QFT in curved spacetime [16, 22, 23]. One could try to deal with Quantum Gravity using the canonical approach, by defining an arrow of time and working on the space-like hypersurfaces perpendicular to it, with equal time commutation relations, but: (i) There are many topologies of the space-time manifold that are not a product $\mathbf{R} \times M_{3}$. (ii) Such non-product topologies are sometimes very interesting. (iii) What does it mean 'equal time' in the presence of Heisenberg's uncertainty principle?

One thus turns naturally towards path-integrals: $<g_{2}, \phi_{2}, \mathscr{S}_{2} \mid g_{1}, \phi_{1}, \mathscr{S}_{1}>=\int \mathscr{D}[g, \phi] e^{i S[g, \phi]}$, where $g_{j}$ denotes the spacetime metric, $\phi_{j}$ are matter fields, $\mathscr{S}_{j}$ general spacetime surfaces $\left(\mathscr{S}_{j}=\right.$ $M_{j} \cup \partial M_{j}$ ), $\mathscr{D}$ a measure over all possible 'paths' leading from the $j=1$ to the $j=2$ values of the intervening magnitudes, and $S$ is the action: $S=\frac{1}{16 \pi G} \int(R-2 \Lambda) \sqrt{-g} d^{4} x+\int L_{m} \sqrt{-g} d^{4} x$, $R$ being the curvature, $\Lambda$ the cc, $g$ the determinant of the metric, and $L_{m}$ the Lagrangian of the matter fields. Stationarity of $S$ under the BCs $\left.\delta g\right|_{\partial M}=0,\left.\vec{n} \cdot \vec{\partial} \delta g\right|_{\partial M}=0$, leads to Einstein's equations: $R_{a b}-\frac{1}{2} g_{a b} R+\Lambda g_{a b}=8 \pi G T_{a b}, T_{a b}$ being the energy-momentum tensor of the matter fields, $T_{a b}=\frac{1}{2 \sqrt{-g}} \frac{\delta L_{m}}{\delta g^{a b}}$. The path-integral formalism provides a way to deal 'perturbatively' with QFT in curved spacetime backgrounds [22]. First, through a rotation in the complex plane one defines an Euclidean action: $i S \longrightarrow-\hat{S}$. One can also easily introduce the finite temperature formalism by the substitution $t_{2}-t_{1}=i \beta$, which yields the partition function $Z=\sum_{n} e^{-\beta E_{n}}$. If one now adheres to the principle that the Feynman propagator is obtained as the limit for $\beta \rightarrow \infty$ of the thermal propagator, we have shown [24] that the usual principal-part prescription in the zeta-function regularization method need not be imposed any more as an additional assumption, since it follows from this more general and natural principle. Next comes the stationary phase approach (also called one-loop or WKB), for calculating the path integral, which consists in expanding around a fixed background: $g=g_{0}+\bar{g}, \phi=\phi_{0}+\bar{\phi}$, and leads to the following expansion in the Euclidean metric: $\hat{S}[g, \phi]=\hat{S}\left[g_{0}, \phi_{0}\right]+S_{2}[\bar{g}, \bar{\phi}]+\cdots$ This is most suitably expressed in terms of determinants (for bosonic, resp. fermionic fields) of the kind (here $A, B$ are the relevant (pseudo)differential operators in the corresponding Lagrangian): $\Delta_{\phi}=\operatorname{det}\left(\frac{1}{2 \pi \mu^{2}} A\right)^{-1}, \Delta_{\psi}=\operatorname{det}\left(\frac{1}{2 \mu^{2}} B\right)$, optimally computed with zeta techniques.

Now, for its application in practice [25], the method of zeta regularization relies on the existence of simple formulas that give the analytic continuation of $\zeta(s)$ from the region of the complex plane extending to the right of the abscissa of convergence, $\operatorname{Re} s>s_{0}$, to the rest of it $[26,27,28]$. These are not only the reflection formula, but also some other expressions, as Jacobi's theta function identity, Poisson's and Plana's resummation formulae, and the Chowla-Selberg formula. But some of these expressions are often restricted to specific cases, and their explicit derivation is usually 
involved [27]. A fundamental property shared by all zeta functions is the existence of a reflection formula. For the Riemann zeta function: $\Gamma(s / 2) \zeta(s)=\pi^{s-1 / 2} \Gamma(1-s / 2) \zeta(1-s)$. For a generic zeta function, $Z(s)$, we may write it as: $Z(\omega-s)=F(\omega, s) Z(s)$. It allows for its analytic continuation in an easy way - what is, in simple cases, the whole story of the zeta function regularization procedure. But the analytically continued expression thus obtained is just another series, which has again a slow convergence behavior, of power series type (the same of the original series, on its convergence domain). S. Chowla and A. Selberg found a formula [29] for the Epstein zeta function in two dimensions, that yields exponentially quick convergence everywhere. In [30] a first attempt was done to try to extend it to inhomogeneous forms; later, to higher dimensions $[19,31]$, both for the homogeneous (quadratic form) and non-homogeneous (quadratic plus affine form) cases. However, some of the new formulas (remarkably the ones corresponding to the zero-mass case, e.g., the original CS framework) were not explicit, and involved solving a non-trivial recurrence, that was solved in [32] and explicit formulas where obtained. Aside from the quadratic case, the linear one is also important for its many applications (system of harmonic oscillators or a multidimensional one). The most general linear zeta function studied is the Barnes' one. Again, many explicit expressions are missing here, as for its derivative in the general case.

Assume the Hamiltonian operator, $H$, has a spectral decomposition of the form (think of a quantum harmonic oscillator): $\left\{\lambda_{i}, \varphi_{i}\right\}_{i \in I}$, being $I$ some set of indices (which can be discrete, continuous, mixed, multiple, ... ). Then, the quantum vacuum energy is obtained as: [27]

$$
E / \mu=\sum_{i \in I}\left\langle\varphi_{i},(H / \mu) \varphi_{i}\right\rangle=\operatorname{Tr}_{\zeta} H / \mu=\sum_{i \in I} \lambda_{i} / \mu=\left.\sum_{i \in I}\left(\lambda_{i} / \mu\right)^{-s}\right|_{s=-1}=\zeta_{H / \mu}(-1),
$$

where $\zeta_{A}$ is the zeta function corresponding to the operator $A$, and the equalities are in the sense of analytic continuation (generically, $A$ is not of the trace class). ${ }^{1}$ Note that the formal sum over the eigenvalues is usually ill defined, and that the last step involves analytic continuation. A regularization parameter $\mu$ with dimensions of mass appears in the process, to render the eigenvalues dimensionless, so that the zeta function can be defined! We shall not discuss these basic details here, which are just at the starting point of the whole renormalization procedure).

\subsection{The Casimir energy}

In fact things do not turn out to be so simple. One cannot assign a meaning to the absolute value of the zero-point energy, and any physical effect is an energy difference between two situations, such as a quantum field in curved space as compared with the same field in flat space, or one satisfying BCs on some surface as compared with the same in its absence, etc. This difference is the Casimir energy: $E_{C}=E_{0}^{B C}-E_{0}=\frac{1}{2}\left(\operatorname{tr} H^{B C}-\operatorname{tr} H\right)$. But here a problem appears. Imposing mathematical boundary conditions (BCs) on physical quantum fields turns out to be a highly nontrivial act. This was discussed in detail in a paper by Deutsch and Candelas [34]. These authors quantized em and scalar fields in the region near an arbitrary smooth boundary, and calculated the renormalized vacuum expectation value of the stress-energy tensor, to find out that the energy

\footnotetext{
${ }^{1}$ The reader should be warned that this $\zeta$-trace is actually no trace in the usual sense. In particular, it is highly non-linear, as often explained by the author elsewhere [33]. Some colleagues are unaware of this fact, which has lead to important mistakes and erroneous conclusions too often.
} 
density diverges as the boundary is approached. Therefore, regularization and renormalization did not seem to cure the problem with infinities in this case and an infinite physical energy was obtained if the mathematical BCs were to be fulfilled. However, the authors argued that surfaces have non-zero depth, and its value could be taken as a handy dimensional cutoff in order to regularize the infinities. Just two years after Deutsch and Candelas' work, Kurt Symanzik carried out a rigorous analysis of QFT in the presence of boundaries [35]. Prescribing the value of the quantum field on a boundary means using the Schrödinger representation, and Symanzik was able to show rigorously that such representation exists to all orders in the perturbative expansion. He showed also that the field operator being diagonalized in a smooth hypersurface differs from the usual renormalized one by a factor that diverges logarithmically when the distance to the hypersurface goes to zero. This requires a precise limiting procedure and point splitting. The issue was proven by him to be meaningful within the domains of renormalized QFT. Here the BCs and the hypersurfaces themselves were treated at a pure mathematical level (zero depth) by using Dirac delta functions.

Recently, a new approach has been postulated [36]. BCs on a field, $\phi$, are enforced on a surface, $S$, by introducing a scalar potential, $\sigma$, of Gaussian shape. When the Gaussian becomes a delta, the BCs (Dirichlet here) are enforced: the delta-shaped potential kills all modes of $\phi$ at the surface. For the rest, the quantum system undergoes a full-fledged QFT renormalization, as in the case of Symanzik's. The results confirm those of [34] in the several models studied albeit they do not seem to agree with those of [35]. They seem to be also in contradiction with the ones quoted in the usual textbooks and review articles dealing with the Casimir effect [37], where no infinite energy density when approaching the Casimir plates has been reported.

\section{On the topology and curvature of space}

The Friedmann-Robertson-Walker (FRW) model, which can be derived as the only family of solutions to the Einstein's equations compatible with the assumptions of homogeneity and isotropy of space, is the generally accepted model of the cosmos. But the FRW is a family with a free parameter, $k$, the curvature, that can be either positive, negative or zero (the flat or Euclidean case). This curvature, or equivalently the curvature radius, $R$, is not fixed by the theory and should be matched with cosmological observations. Moreover, the FRW model, and Einstein's equations themselves, can only provide local properties, not global ones, so they cannot tell about the overall topology of our world: is it closed or open? finite or infinite? Even being quite clear that it is, in any case, extremely large — and possibly the human species will never reach more than an infinitesimally tiny part of it - the question is very appealing to any (note that this discussion concerns only three-dimensional space curvature and topology, time will not be involved).

\subsection{On the curvature}

Serious attempts to measure the possible curvature of the space we live in go back to Gauss, who measured the sum of the three angles of a big triangle with vertices on the picks of three far away mountains (Brocken, Inselberg, and Hohenhagen). He was looking for evidence that the geometry of space is non-Euclidean. The idea was brilliant, but condemned to failure: one needs a much bigger triangle to try to find the possible non-zero curvature of space. Now cosmologist have recently measured the curvature radius $R$ by using the largest triangle available, namely one 
with us at one vertex and with the other two on the hot opaque surface of the ionized hydrogen that delimits our visible universe and emits the CMB radiation (some 3 to $4 \times 10^{5}$ years after the Big Bang) [38]. The CMB maps exhibit hot and cold spots. It can be shown that the characteristic spot angular size corresponds to the first peak of the temperature power spectrum, which is reached for an angular size of $.5^{\circ}$ (approximately the one subtended by the Moon) if space is flat. If it has a positive curvature, spots should be larger (with a corresponding displacement of the position of the peak), and correspondingly smaller for negative curvature. The joint analysis of the considerable amount of data obtained during the last years by balloon experiments (BOOMERanG, MAXIMA, DASI) [39, 40], combined with galaxy clustering data, produced a lower bound for $|R|>20 h^{-1} \mathrm{Gpc}$, i.e. twice as large as the radius of the observable universe, of about $R_{U} \simeq 9 h^{-1} \mathrm{Gpc}$.

\subsection{On the topology}

Let us repeat that GR does not prescribe the topology of the universe, or its being finite or not, and the universe could perfectly be flat and finite. The simplest non-trivial model from the theoretical viewpoint is the toroidal topology. Traces for this and more elaborated ones, as negatively curved but compact spaces, have been profusely investigated, and some circles in the sky with near identical temperature patterns were identified [41]. And yet more papers appear from time to time proposing a new topology [42]. However, to summarize all these efforts and the observational situation, and once the numerical data are interpreted without bias (what sometimes was not the case, and led to erroneous conclusions), it seems at present that available data point towards a very large (we may call it infinite) flat space.

\section{Vacuum energy fluctuations and the cosmological constant}

The issue of the cc has got renewed thrust from the observational evidence of an acceleration in the expansion of our Universe, initially reported by two different groups [43]. There was some controversy on the reliability of the results obtained from those observations and on its precise interpretation, by a number of different reasons. Anyway, after new data has been gathered, there is now consensus among the community of cosmologists that, in fact, an acceleration is there, and that it has the order of magnitude obtained in the above mentioned observations $[44,45,46]$. As a consequence, many theoreticians have urged to try to explain this fact, and also to try to reproduce the precise value of the cc coming from these observations [47, 48, 49].

Now, as crudely stated by Weinberg [50], it is even more difficult to explain why the cc is so small but non-zero, than to build theoretical models where it exactly vanishes [51]. Rigorous calculations performed in quantum field theory on the vacuum energy density, $\rho_{V}$, corresponding to quantum fluctuations of the fields we observe in nature, lead to values that are over 120 orders of magnitude in excess of those allowed by observations of the space-time around us. Energy always gravitates, therefore the energy density of the vacuum, more precisely, the vacuum expectation value of the stress-energy tensor $\left\langle T_{\mu \nu}\right\rangle \equiv-\mathscr{E} g_{\mu \nu}$ appears on the rhs of Einstein's equations: $R_{\mu \nu}-$ $\frac{1}{2} g_{\mu \nu} R=-8 \pi G\left(\tilde{T}_{\mu \nu}-\mathscr{E} g_{\mu \nu}\right)$. It affects cosmology: $\tilde{T}_{\mu \nu}$ contains excitations above the vacuum, and is equivalent to a $c c \lambda=8 \pi G \mathscr{E}$. Recent observations yield [52]

$$
\lambda_{\text {obs }}=\left(2.14 \pm 0.13 \times 10^{-3} \mathrm{eV}\right)^{4} \sim 4.32 \times 10^{-9} \mathrm{erg} / \mathrm{cm}^{3}
$$


It is an old idea that the cc gets contributions from zero point fluctuations [2]

$$
E_{0}=\frac{\hbar c}{2} \sum_{n} \omega_{n}, \quad \omega=k^{2}+m^{2} / \hbar^{2}, k=2 \pi / \lambda .
$$

Evaluating in a box and putting a cut-off at maximum $k_{\max }$ corresponding to reliable QFT physics (e.g., the Planck energy): $\rho \sim \hbar k_{\text {Planck }}^{4} / 16 \pi^{2} \sim 10^{123} \rho_{\text {obs. }}$.

Assuming one will be able to prove (in the future) that the ground value of the $\mathrm{cc}$ is zero (as many suspected until recently), we will be left with this incremental value coming from the topology or BCs. This sort of two-step approach to the cc is becoming more and more popular recently as a way to try to solve this very difficult issue [3]. We have seen, using different examples, that this value acquires in fact the correct order of magnitude - corresponding to the one coming from the observed acceleration in the expansion of our universe- under some reasonable conditions. We put forward a quite simple and primitive idea (but, for the same reason, of possibly far reaching consequences), related with the global topology of the universe [53] and in connection with the possibility that a faint scalar field pervading the universe could exist. Fields of this kind are ubiquitous in inflationary models, quintessence theories, and the like. In other words, we do not pretend to solve the old problem of the cc, not even to contribute significantly to its understanding, but just to present simple and usual models which show that the right order of magnitude of (some contributions to) $\rho_{V}$ which lie in the precise range deduced from the astrophysical observations are not difficult to get. To say it in different words, we only address here the 'second stage' of what has been termed by Weinberg [50] the new cc problem.

\section{Vacuum energy contribution in different models}

\subsection{Simple model with large and small compactified dimensions}

We assume the existence of a scalar field extending through the universe and calculate the contribution to the $\mathrm{cc}$ from the Casimir energy density of this field, for some typical BCs. Ultraviolet contributions will be safely set to zero by some mechanism of a fundamental theory. Another hypothesis will be the existence of both large and small dimensions (the total number of large spatial coordinates being always three), some of which may be compactified, so that the global topology of the universe will play an important role. There is a quite extensive literature both in the subject of what is the global topology of spatial sections of the universe [53] and also on the issue of the possible contribution of the Casimir effect as a source of some sort of cosmic energy, as in the case of the creation of a neutron star [54]. There are arguments that favor different topologies, as a compact hyperbolic manifold for the spatial section, what would have clear observational consequences [55]. Other interesting work along these lines was reported in [25] and related ideas have been discussed very recently in [56]. However, we differ from all those in that emphasis is put now in obtaining the right order of magnitude for the effect. At the present stage it has no sense to consider the whole amount of possibilities concerning the nature of the field, the different models for the topology of the universe, and the different BCs possible, with its effect on the sign of the force too. This is left to a second, more detailed analysis. From previous results [27] we know that the range of orders of magnitude of the vacuum energy density for the most common possibilities is not so widespread, and may only differ by at most a couple of digits. This will allow us, both for 
the sake of simplicity and universality, to deal with two simple situations, corresponding to a scalar field with periodic BCs or spherically compactified. As explained in [57], most cases with usual $\mathrm{BCs}$ reduce to those, from a mathematical viewpoint.

Consider a universe with a space-time of one of the following types: $\mathbb{R}^{d+1} \times \mathbb{T}^{p} \times \mathbb{T}^{q}, \mathbb{R}^{d+1} \times$ $\mathbb{T}^{p} \times \mathbb{S}^{q}, \ldots$, which are actually plausible models for the space-time topology. Here, $d \geq 0$ stands for a possible number of non-compactified dimensions. Recall the physical contribution to the vacuum or zero-point energy $<0|H| 0>$ is obtained by subtracting the vacuum energy corresponding to the situation with the only change that compactification is absent (in practice this is done by conveniently sending the compactification radii to infinity). As well known, both of these vacuum energies are in fact infinite, but it is its difference $E_{C}=\left\langle 0|H| 0>\left.\right|_{R}-<0|H| 0>\left.\right|_{R \rightarrow \infty}(R\right.$ a typical compactification length) that makes physical sense, giving rise to the finite value of the Casimir energy $E_{C}$. Renormalization must be then carried out. In fact we will discuss the Casimir (or vacuum) energy density, $\rho_{C}=E_{C} / V$, which can account for either a finite or an infinite volume of the spatial section of the universe (from now on we shall assume that all diagonalizations already correspond to energy densities, volume factors are replaced at the end). In terms of the spectrum $\left\{\lambda_{n}\right\}$ of $H$ : $<0|H| 0>=\frac{1}{2} \sum_{n} \lambda_{n}$, where the sum over $n$ is over the whole spectrum, which may involve several continuum and several discrete indices. The last appear tipically when compactifying the space coordinates (much as time compactification gives rise to a finite-temperature field theory), as in the cases we are going to consider. Thus, integration over $d$ continuous dimensions and multiple summations over $p+q$ indices appear (for a pedagogical description, see [57]).

The physical vacuum energy density in our case, where the contribution of a scalar field $\phi$ living in a partly compactified spatial section of the universe is considered, with

$$
S=\frac{1}{2} \int d^{4} x \sqrt{-g}\left[g^{\mu v} \partial_{\mu} \phi \partial_{v} \phi+\left(m^{2}+\xi R\right) \phi^{2}\right]
$$

will be denoted by $\rho_{\phi}$ (this is just the contribution to $\rho_{V}$ coming from this field, there might be other, in general), $\rho_{\phi}=\frac{1}{2} \sum_{i} \lambda_{i}=\frac{1}{2} \sum_{\mathbf{k}} \frac{1}{\mu}\left(k^{2}+M^{2}\right)^{1 / 2}$, where the sum $\sum_{\mathbf{k}}$ is a generalized one, $M^{2}=m^{2}+\xi R$ is an effective mass term, and $\mu$ is the usual mass-dimensional parameter to render the eigenvalues dimensionless (the renormalization parameter; we take $\hbar=c=1$ ). The mass $m$ of the field will be here considered to be arbitrarily small but different from zero, for now, for computational reasons - as well as for physical ones, since a very tiny mass for the field can never be excluded $[58,59]$. Our model is stationary, while the universe is expanding. A more careful calculation shows that this effect can actually be dismissed at the level of order of magnitude, since its value cannot surpass the one that we will get (as is seen from the present value of the expansion rate $\Delta R / R \sim 10^{-10}$ per year or from direct consideration of the Hubble coefficient). Recent considerations on the dynamical Casimir effect may be important in a future, more detailed analysis [60]. For simplicity we perform a static calculation. As a consequence, the values obtained correspond to the present epoch.

Let us write down in detail the formulas corresponding to the two first topologies, as described 
above. For a $(p, q)$-toroidal universe, with $p$ the number of 'large' and $q$ of 'small' dimensions:

$$
\begin{gathered}
\rho_{\phi}=\frac{\pi^{-d / 2}}{2^{d} \Gamma(d / 2) \prod_{j=1}^{p} a_{j} \prod_{h=1}^{q} b_{h}} \int_{0}^{\infty} d k k^{d-1} \sum_{\mathbf{n}_{p}=-\infty}^{\infty} \sum_{\mathbf{m}_{q}=-\infty}^{\infty}\left[\sum_{j=1}^{p}\left(\frac{2 \pi n_{j}}{a_{j}}\right)^{2}+\sum_{h=1}^{q}\left(\frac{2 \pi m_{h}}{b_{h}}\right)^{2}+M^{2}\right]^{1 / 2} \\
\sim \frac{1}{a^{p} b^{q}} \sum_{\mathbf{n}_{p}, \mathbf{m}_{q}=-\infty}^{\infty}\left(\frac{1}{a^{2}} \sum_{j=1}^{p} n_{j}^{2}+\frac{1}{b^{2}} \sum_{h=1}^{q} m_{h}^{2}+M^{2}\right)^{(d+1) / 2+1}
\end{gathered}
$$

where the last formula corresponds to the case when all large (resp. all small) compactification scales are the same. In this last expression the squared mass of the field should be divided by $4 \pi^{2} \mu^{2}$, but we have renamed it again $M^{2}$ to simplify the ensuing formulas. We also will not take care for the moment of the mass-dim factor $\mu$ in other places since formulas would get unnecessarily complicated and there is no problem in recovering it at the end of the calculation. For a ( $p$-toroidal, $q$-spherical)-universe,

$$
\begin{gathered}
\rho_{\phi}=\frac{\pi^{-d / 2}}{2^{d} \Gamma(d / 2) \prod_{j=1}^{p} a_{j} b^{q}} \int_{0}^{\infty} d k k^{d-1} \sum_{\mathbf{n}_{p}=-\infty}^{\infty} \sum_{l=1}^{\infty} P_{q-1}(l)\left[\sum_{j=1}^{p}\left(\frac{2 \pi n_{j}}{a_{j}}\right)^{2}+\frac{Q_{2}(l)}{b^{2}}+M^{2}\right]^{1 / 2} \\
\sim \frac{1}{a^{p} b^{q}} \sum_{\mathbf{n}_{p}=-\infty}^{\infty} \sum_{l=1}^{\infty} P_{q-1}(l)\left(\frac{4 \pi^{2}}{a^{2}} \sum_{j=1}^{p} n_{j}^{2}+\frac{l(l+q)}{b^{2}}+M^{2}\right)^{(d+1) / 2+1},
\end{gathered}
$$

where $P_{q-1}(l)$ is a polynomial in $l$ of degree $q-1$. On dealing with our observable universe, we assume that $d=3-p$, the number of non-compactified, 'large' spatial dimensions (thus, no $d$ dependence will remain). All these expressions for $\rho_{\phi}$ need to be regularized and we use the zeta function method, as previously explained.

We will use zeta function regularization, taking advantage of the very powerful equalities that have been derived by the author [19, 24], and which reduce the enormous burden of such computations to the easy application of some formulas. For the sake of completeness, let us very briefly summarize how this works $[61,57]$. We deal here only with the case when the spectrum of the Hamiltonian operator is known explicitly. Going back to the most general expressions of the Casimir energy corresponding to this case, we replace the exponents in them with a complex variable, $s$, thus obtaining the zeta function associated with the operator as:

$$
\zeta(s)=\frac{1}{2} \sum_{\mathbf{k}}\left(\frac{k^{2}+M^{2}}{\mu^{2}}\right)^{-s / 2} .
$$

The next step is to perform the analytic continuation of the zeta function from a domain of the complex s-plane with Re $s$ big enough (where it is perfectly defined by this sum) to the point $s=-1$, to obtain: $\rho_{\phi}=\zeta(-1)$. The effectiveness of this method has been sufficiently described before (see, e.g., [27]). As we know from precise Casimir calculations in those references, no further subtraction or renormalization is needed in the cases here considered, in order to obtain the physical value for the vacuum energy density (there is actually a subtraction at infinity taken into account, as carefully described above, but it is of null value, and no renormalization, not even a finite one, very common to other frameworks, applies here).

Using the formulas [19] that generalize the well-known Chowla-Selberg expression to the situations considered above, Eqs. (5.2) and (5.3) — namely, multidimensional, massive cases — we 
can provide arbitrarily accurate results for different values of the compactification radii. However, as argued above we only aim here at matching the order of magnitude of the Casimir value and, thus, we shall just deal with the most simple cases of Eqs. (5.2) or (5.3), which yield the same orders of magnitude as the rest of them). Also in accordance with this observation, we notice that among the models here considered and which lead to the values that will be obtained below, there are in particular the very important typical cases of isotropic universes with the spherical topology. As all our discussion here is in terms of orders of magnitude and not of precise values with small errors, all these cases are included on equal footing. But, on the other hand, it has no sense to present a lengthy calculation dealing in detail with all the possible spatial geometries. Anyhow, all these calculations can indeed be done, and are very similar to the one here, as has been described in detail elsewhere [25, 27].

For the analytic continuation of the zeta function corresponding to (5.2), we obtain:[19]

$$
\begin{aligned}
\zeta(s)= & \frac{2 \pi^{s / 2+1}}{a^{p-(s+1) / 2} b^{q-(s-1) / 2} \Gamma(s / 2)} \sum_{\mathbf{m}_{q}=-\infty}^{\infty} \sum_{h=0}^{p}\left(\begin{array}{l}
p \\
h
\end{array}\right) 2^{h} \sum_{\mathbf{n}_{h}=1}^{\infty}\left(\frac{\sum_{j=1}^{h} n_{j}^{2}}{\sum_{k=1}^{q} m_{k}^{2}+M^{2}}\right)^{(s-1) / 4} \\
& \times K_{(s-1) / 2}\left[\frac{2 \pi a}{b} \sqrt{\sum_{j=1}^{h} n_{j}^{2}\left(\sum_{k=1}^{q} m_{k}^{2}+M^{2}\right)}\right]
\end{aligned}
$$

where $K_{v}(z)$ is the modified Bessel function of the second kind. Having performed already the analytic continuation, this expression is ready for the substitution $s=-1$, and yields

$$
\rho_{\phi}=-\frac{1}{a^{p} b^{q+1}} \sum_{h=0}^{p}\left(\begin{array}{l}
p \\
h
\end{array}\right) 2^{h} \sum_{\mathbf{n}_{h}=1}^{\infty} \sum_{\mathbf{m}_{q}=-\infty}^{\infty} \sqrt{\frac{\sum_{k=1}^{q} m_{k}^{2}+M^{2}}{\sum_{j=1}^{h} n_{j}^{2}}} K_{1}\left[\frac{2 \pi a}{b} \sqrt{\sum_{j=1}^{h} n_{j}^{2}\left(\sum_{k=1}^{q} m_{k}^{2}+M^{2}\right)}\right]
$$

Now, from the behaviour of the function $K_{v}(z)$ for small values of its argument, $K_{v}(z) \sim \frac{1}{2} \Gamma(v)(z / 2)^{-v}$, $z \rightarrow 0$, we obtain, in the case when $M$ is not large,

$$
\begin{aligned}
\rho_{\phi}= & -\frac{1}{a^{p} b^{q+1}}\left\{M K_{1}\left(\frac{2 \pi a}{b} M\right)+\sum_{h=0}^{p}\left(\begin{array}{l}
p \\
h
\end{array}\right) 2^{h} \sum_{\mathbf{n}_{h}=1}^{\infty} \frac{M}{\sqrt{\sum_{j=1}^{h} n_{j}^{2}}}\right. \\
& \left.\times K_{1}\left(\frac{2 \pi a}{b} M \sqrt{\sum_{j=1}^{h} n_{j}^{2}}\right)+\mathscr{O}\left[q \sqrt{1+M^{2}} K_{1}\left(\frac{2 \pi a}{b} \sqrt{1+M^{2}}\right)\right]\right\} .
\end{aligned}
$$

The only presence of the mass-dim parameter $\mu$ is as $M / \mu$ everywhere. This does not affect the small- $M$ limit, $M / \mu<<b / a$. Inserting now in the expression the $\hbar$ and $c$ factors, we finally get

$$
\rho_{\phi}=-\frac{\hbar c}{2 \pi a^{p+1} b^{q}}\left[1+\sum_{h=0}^{p}\left(\begin{array}{l}
p \\
h
\end{array}\right) 2^{h} \alpha\right]+\mathscr{O}\left[q K_{1}\left(\frac{2 \pi a}{b}\right)\right]
$$

where $\alpha$ is some finite, computable constant obtained as an explicit geometric sum in the limit $M \rightarrow 0$. It is remarkable that we obtain a well defined limit, independent of $M^{2}$, provided $M^{2}$ is not large: a physically nice situation turns out to correspond to the mathematically rigorous case. This is the expression one gets not just for the model considered, but for many cases, corresponding to 
different fields, topologies, and $\mathrm{BCs}$ — aside from the sign in front of the formula, that may change with the number of compactified dimensions and the nature of the BCs (in particular, for Dirichlet BCs one gets a value in the same order of magnitude but of opposite sign).

\subsection{Numerical results}

For the most common variants, the constant $\alpha$ in (5.8) has been calculated to be of order $10^{2}$, and the whole factor, in brackets, of the first term in (5.8) has a value of order $10^{7}$. This shows the value of a precise calculation, as the one undertaken here, together with the fact that just a naive consideration of the dependencies of $\rho_{\phi}$ on the powers of the compactification radii, $a$ and $b$, is not enough in order to obtain the correct result. Notice, moreover, the non-trivial change in the power dependencies from going from Eq. (5.7) to Eq. (5.8).

For the compactification radii at small scales, $b$, we shall simply take the magnitude of the Planck length, $b \sim l_{P(\text { lanck })}$, while the typical value for the large scales, $a$, will be the present size of the observable universe, $a \sim R_{U}$. With this choice, the order of the quotient $a / b$ in the argument of $K_{1}$ is $a / b \sim 10^{60}$. Thus, we see immediately that, in fact, the final expression for the vacuum energy density is completely independent of the mass $M$ of the field, provided this is very small (eventually zero). In fact, since the last term in Eq. (5.8) is exponentially vanishing, for large arguments of the Bessel function $K_{1}$, this contribution is zero, for all practical purposes, what is already a very nice result. Taken in ordinary units (and after tracing back all the transformations suffered by the mass term $M$ ) the actual bound on the mass of the scalar field is $M \leq 1.2 \times 10^{-32}$ $\mathrm{eV}$, that is, physically zero, since it is lower by several orders of magnitude than any bound coming from the more usual SUSY theories - where in fact scalar fields with low masses of the order of that of the lightest neutrino do show up [48], which may have observable implications.

\begin{tabular}{|c||c|c|c|c|}
\hline \hline$\rho_{\phi}$ & $p=0$ & $p=1$ & $p=2$ & $p=3$ \\
\hline \hline$b=l_{P}$ & $10^{-13}$ & $10^{-6}$ & 1 & $10^{5}$ \\
\hline$b=10 l_{P}$ & $10^{-14}$ & {$\left[10^{-8}\right]$} & $10^{-3}$ & 10 \\
\hline$b=10^{2} l_{P}$ & $10^{-15}$ & {$\left[10^{-10}\right]$} & $10^{-6}$ & $10^{-3}$ \\
\hline$b=10^{3} l_{P}$ & $10^{-16}$ & $10^{-12}$ & {$\left[10^{-9}\right]$} & $\left(10^{-7}\right)$ \\
\hline$b=10^{4} l_{P}$ & $10^{-17}$ & $10^{-14}$ & $10^{-12}$ & $\left(10^{-11}\right)$ \\
\hline$b=10^{5} l_{P}$ & $10^{-18}$ & $10^{-16}$ & $10^{-15}$ & $10^{-15}$ \\
\hline \hline
\end{tabular}

Table 1: Orders of magnitude of the vacuum energy density contribution, $\rho_{\phi}$, of a massless scalar field to the cc, $\rho_{V}$, for $p$ large compactified dimensions and $q=p+1$ small compactified dimensions, $p=0, \ldots, 3$, for different values of the small compactification length, $b$, proportional to the Planck length $l_{P}$. In brackets, values that better match the observed cc result, and in parenthesis the otherwise closest approximations.

By replacing all these values in Eq. (5.8), we obtain the results listed in Table 1, for the orders of magnitude of the vacuum energy density corresponding to a sample of different numbers of compactified (large and small) dimensions and for different values of the small compactification length in terms of the Planck length. Notice again that the total number of large space dimensions is three, as corresponds to our observable universe. As we see from Table 1, good coincidence with the observational value for the $\mathrm{cc}$ is obtained for the contribution of a massless scalar field, $\rho_{\phi}$, for $p$ large compactified dimensions and $q=p+1$ small compactified dimensions, $p=0, \ldots, 3$, and 
this for values of the small compactification length, $b$, of the order of 100 to 1000 times the Planck length $l_{P}$. Full agreement is obtained only for cases where there is exactly one small compactified dimension in excess of the number of large compactified dimensions. We must point out that the $p$ large and $q$ small dimensions are not all that are supposed to exist (in that case $p$ should be at least, and at most, 3 and the other cases would lack any physical meaning). In fact, as we have pointed out before, $p$ and $q$ refer to the compactified dimensions only, but there may be other, noncompactifed dimensions (exactly $3-p$ in the case of the 'large' ones), what translates into a slight modification of the formulas above, but does not change the order of magnitude of the final numbers obtained, assuming the most common BCs for the non-compactified dimensions (see e.g. [27] for an explanation of this technical point). In particular, the cases of pure spherical compactification and of mixed toroidal (for small magnitudes) and spherical (for big ones) compactification can be treated in this way and yield results in the same order of magnitude range. Both these cases correspond to (observational) isotropic spatial geometries. Also to be remarked again is the nontriviality of these calculations, when carried out exactly, as done here, to the last expression, what is apparent from the use of the generalized Chowla-Selberg formula. Simple power counting is unable to provide the correct order of magnitude of the results.

Dimensionally speaking, within the global approach adopted in the present paper everything is dictated, in the end, by the two basic lengths in the problem, which are its Planck value and the radius of the observable Universe. Just by playing with these numbers in the context of this precise calculation of the Casimir effect, we have shown that the observed value of $\rho_{V}$ may be remarkably well fitted, under general hypothesis, for the most common models of the space-time topology. Notice also that the most precise fits with the observational value of the cc are obtained for $b$ between $b=100 l_{P}$ and $b=1000 l_{P}$, with $(1,2)$ and $(2,3)$ compactified dimensions, respectively. The fact that the value obtained for the cc is so sensitive to the input may be viewed as a drawback but also, on the contrary, as a very positive feature of our model. For one, the Table 1 has a sharp discriminating power. In other words, there is in fact no tuning of a 'free parameter' in our model and the number of large compactified dimensions could have been fixed beforehand, to respect what we know already of our observable universe.

Also, it proves that the observational value is not easy at all to obtain. Table 1 itself proves that there is only very little chance of getting the right figure (a truly narrow window, since very easily we are off by several orders of magnitude). In fact, if we trust this value with the statistics at hand, we can undoubtedly claim - through use of the model- that the ones so clearly picked up by Table 1 are the only two possible configurations of our observable universe (together with a couple more coming from corresponding spherical compactifications). And all them correspond to a marginally closed universe, in full agreement too with other completely independent analysis of the observational data $[45,43]$.

Many questions may be posed to the simple models presented here, as concerning the dynamics of the scalar field, its couplings with gravity and other fields, a possible non-symmetrical behaviour with respect to the large and small dimensions, or the relevance of vacuum polarization (see [62] concerning this last point). Above we have already argued that they can be proven to have little influence on the final numerical result (cf., in particular, the mass obtained for the scalar field in [58], extremely close to our own result, and the corresponding discussion there). From the very existence and specific properties of the cosmic microwave radiation (CMB) -which 
mimics somehow the situation described (the 'mass' corresponding to the CMB is also in the sublightest-neutrino range) - we are led to the conclusion that such a field could be actually present, unnoticed, in our observable universe. In fact, the existence of scalar fields of very low masses is also demanded by other frameworks, as SUSY models, where the scaling behaviour of the cc has been considered [48].

Let us finally recall again that the Casimir effect is an ubiquitous phenomena. Its contribution may be small (as seems to be the case, yet controverted, to sonoluminiscence), or of some 10-30\% (the right order of magnitude, as in wetting phenomena involving $\mathrm{He}$ in condensed matter physics). Here we have seen that it is of the right order of magnitude, corresponding to our present epoch in the evolution of the universe. The implication that this calculation may bear for the early universe and inflation is not clear from the final result, since it should be adapted to the situation and BCs corresponding to those primeval epochs, what cannot be seen straightforwardly.

Acknowledgments. This work has been supported in part by MEC (Spain), projects BFM200300620 and PR2006-0145, and by AGAUR (Generalitat de Catalunya), contract 2005SGR-00790.

\section{References}

[1] S.W. Hawking, Commun. Math. Phys. 55, 133 (1977); S.W. Hawking and W. Israel, Eds., "General Relativity, an Einstein Centenary Survey” (Cambridge University Press, 1979).

[2] Ya.B. Zeldovich, Soviet Physics Uspekhi 11, 382 (1968) [Uspekhi Fiz. Nauk, 95, 209 (1968)].

[3] C.P. Burgess et al., hep-th/0606020, 0510123; T. Padmanabhan, gr-qc/0606061; L. Parker and A. Raval, Phys. Rev. D62, 083503 (2000); Phys. Rev. Lett. 86, 749 (2001).

[4] A.P. Calderón and A. Zygmund, Am. J. Math. 79, 801 (1957); Studia Math. 20, 171 (1961); A.P. Calderón and R. Vaillancourt, Proc. Nat. Acad. Sci. U.S.A. 69, 1185 (1972).

[5] M. Atiyah and I.M. Singer, Ann. Math. 87, 484 and 546 (1968); 93, 119 and 139 (1971).

[6] R.T. Seeley, Am. Math. Soc. Proc. Symp. Pure Math. 10, 288 (1967); Am. J. Math. 91, 889 (1969).

[7] L. Hörmander, The analysis of partial differential operators, Vols I-IV (Springer, Berlin, 1983-85); F. Treves, Introduction to pseudodifferential and Fourier integral operators, Vols. I and II (Plenum, New York, 1980); M.E. Taylor, Pseudodifferential operators (Princeton Univ. Press, 1981); H. Lawson and M.L. Michelsohn, Spin geometry (Princeton Univ. Press, 1989).

[8] M. Kontsevich and S. Vishik, Functional Analysis on the Eve of the 21st Century. Vol. 1, 173 (1993).

[9] D.B. Ray, Adv. in Math. 4, 109 (1970); D.B. Ray and I.M. Singer, Adv. in Math. 7, 145 (1971); Ann. Math. 98, 154 (1973).

[10] J. Collins, Renormalization: An Introduction to Renormalization, the Renormalization Group and the Operator-Product Expansion (Cambridge University Press, 1984); W. D. McComb, Renormalization Methods: A Guide for Beginners (Oxford University Press, 2004).

[11] A. Connes, Noncommutative Geometry (Academic Press, New York, 1994).

[12] M. Wodzicki. Noncommutative Residue, Chapter I, in Lecture Notes in Mathematics, Yu.I. Manin, editor, Vol. 1289, 320 (Springer-Verlag, Berlin, 1987).

[13] E. Elizalde, J.Phys. A30, 2735 (1997) 
[14] C.Kassel, Asterisque 177, 199 (1989), Sem. Bourbaki.

[15] E. Elizalde, L. Vanzo and S. Zerbini, Commun. Math. Phys. 194, 613 (1998); E. Elizalde, A. Filippi, L. Vanzo and S. Zerbini, D57, 7430 (1998).

[16] P. Ramond, Field Theory: a Modern Primer (Addison-Wesley, Redwood City, 1989).

[17] N. Evans, Phys. Lett. B457, 127 (1999); J.S. Dowker, On the relevance on the multiplicative anomaly hep-th/9803200; E. Elizalde, A. Filippi, L. Vanzo and S. Zerbini, Is the multiplicative anomaly dependent on the regularization? hep-th/9804071; E. Elizalde, A. Filippi, L. Vanzo and S. Zerbini, Is the multiplicative anomaly relevant? hep-th/9804072.

[18] E. Elizalde, G. Cognola and S. Zerbini, Nucl. Phys. B532, 407 (1998); J.J. McKenzie-Smith and D.J. Toms, Phys. Rev. 558105001 (1998); A.A. Bytsenko and F.L. Williams, J. Math. Phys. 39, 1075 (1998).

[19] E. Elizalde, Commun. Math. Phys. 198, 83 (1998).

[20] G. H. Hardy, Divergent Series (Oxford University Press, 1949).

[21] T. Kato, Perturbation Theory for Linear Operators (Springer, Berlin, 1980)

[22] N. Birrell and P.C.W. Davies, "Quantum Fields in Curved Spaces” (Cambridge University Press, Cambridge, 1982).

[23] I.L. Buchbinder, S.D. Odintsov and I.L. Shapiro, "Effective Action in Quantum Gravity" (IOP Publishing, Bristol, 1992).

[24] K. Kirsten and E. Elizalde, Phys. Lett. B365, 72 (1995).

[25] E. Elizalde, J. Math. Phys. 35, 6100 (1994); 35, 3308 (1994).

[26] E. Elizalde, J. Phys. A22, 931 (1989); E. Elizalde and A. Romeo, Phys. Rev. D40, 436 (1989); E. Elizalde, J. Math. Phys. 31, 170 (1990).

[27] E. Elizalde, S.D. Odintsov, A. Romeo, A.A. Bytsenko and S. Zerbini, Zeta regularization techniques with applications (World Sci., Singapore, 1994); E. Elizalde, Ten Physical Applications of Spectral Zeta Functions (Springer-Verlag, Berlin, 1995); A.A. Bytsenko, G. Cognola, L. Vanzo and S. Zerbini, Phys. Reports 266, 1 (1996); K. Kirsten, Spectral functions in mathematics and physics (Chapman \& Hall, London, 2001); A.A. Bytsenko, G. Cognola, E. Elizalde, V. Moretti and S. Zerbini, Analytic Aspects of Quantum Fields (World Sci., Singapore, 2004).

[28] E. Elizalde, J. Phys. A18, 1637 (1985); E. Elizalde, Math. of Computation 47, 175 (1986); E. Elizalde and A. Romeo, Int. J. Math. and Math. Sci. 13, 453 (1990).

[29] S. Chowla and A. Selberg, Proc. Nat. Acad. Sci. U.S.A. 35 (1949) 317.

[30] E. Elizalde, J. Phys. A27 (1994) 3775.

[31] E. Elizalde, J. Comput. Appl. Math. 118, 125 (2000); A.A. Bytsenko, E. Elizalde, and S. Zerbini, Phys. Rev. D64, 105024 (2001).

[32] E. Elizalde, J. Phys. A34, 3025 (2001).

[33] E. Elizalde, J. High Energy Phys. 9907, 015 (1999).

[34] D. Deutsch and P. Candelas, Phys. Rev. D20, 3063 (1979).

[35] K. Symanzik, Nucl. Phys. B190, 1 (1981). 
[36] R. L. Jaffe, Unnatural acts: unphysical consequences of imposing boundary conditions on quantum fields, hep-th/0307014; N. Graham, R. L. Jaffe, V. Khemani, M. Quandt, M. Scandurra and H. Weigel, Phys. Lett. B572, 196 (2003); N. Graham, R. L. Jaffe, V. Khemani, M. Quandt, M. Scandurra and H. Weigel, Nucl. Phys. B645, 49 (2002); N. Graham, R. L. Jaffe and H. Weigel, Int. J. Mod. Phys. A17, 846 (2002).

[37] V.M. Mostepanenko and N.N. Trunov, The Casimir effect and its application (Clarendon Press, Oxford, 1997); K.A. Milton, The Casimir Effect: Physical manifestations of zero-point energy (World Scientific, Singapore, 2001); M. Bordag, U. Mohideen and V.M. Mostepanenko, Phys. Rept. 353, 1 (2001).

[38] M. Tegmark, Science, 296, 1427 (2002).

[39] P. de Bernardis et al., Nature 404, 955 (2000).

[40] S. Hanany et al., Astrophys. J. 545, L5 (2000); A. Balbi et al., Astrophys. J. 545, L1 (2000); erratum-ibid. 558, L145 (2001).

[41] N.J. Cornish et al., Phys. Rev. Lett. 92, 201302 (2004).

[42] J.-P. Luminet et al., Nature, 425, 593 (2003).

[43] S. Perlmutter et al. [Supernova Cosmology Project Collab.], Astrophys. J. 517, 565 (1999); A.G. Riess et al. [Hi-Z Supernova Team Collab.], Astron. Journ. 116, 1009 (1998).

[44] A.G. Riess, Publ. Astron. Soc. Pac. 112, 1284 (2000).

[45] S.M. Carroll, Living Rev. Rel. 4, 1 (2001).

[46] S.M. Carroll, Why is the Universe Accelerating?, Contribution to Measuring and Modeling the Universe, Carnegie Observatories Astrophysics Series Vol. 2, ed. W. L. Freedman.

[47] V. Sahni and A. Starobinsky, Int. J. Mod. Phys. D9, 373 (2000).

[48] I.L. Shapiro and J. Solà, Phys. Lett. B475, 236 (2000).

[49] T.R. Mongan, Gen. Rel. Grav. 33, 1415 (2001).

[50] S. Weinberg, Phys. Rev. D61 103505 (2000).

[51] S. Weinberg, Rev. Mod. Phys. 61, 1 (1989); W. Fischler, I. Klebanov, J. Polchinski, and L. Susskind, Nucl. Phys. B237, 157 (1989); S. Coleman, Nucl. Phys. B310, 643 (1988); S. Coleman, Nucl. Phys. B307, 867 (1988); S. Weinberg, Phys. Rev. Lett. 59, 2607 (1987); E. Baum, Phys. Lett. B133, 185 (1984); S. W. Hawking, in Shelter Island II - Proceedings of the 1983 Shelter Island Conference on Quantum Field Theory and the Fundamental Problems of Physics, ed. R. Jackiw et al. (MIT Press, Cambridge, 1995); Phys. Lett. B134, 403 (1984).

[52] M. Tegmark et al. [SDSS Collab.], Phys. Rev. D69, 103501 (2004); D.J. Eisenstein et al., Astrophys. J. 633, 560 (2005); J.K. Adelman-McCarthy et al., Astrophys. J. Suppl. 162, 38 (2006).

[53] V. Blanloeil and B.F. Roukema, Eds., Cosmological Topology in Paris 1998 [astro-ph/0010170]. See also the entire Vol. 15 of Classical and Quantum Gravity (1998).

[54] I.Yu. Sokolov, JETP Lett. 57, 617 (1993).

[55] N.J. Cornish, D. Spergel, and G. Starkman, Class. Quant. Grav. 15, 2657 (1998); ibid. Phys. Rev. Lett. 77, 215 (1996); D. Müller, H.V. Fagundes, and R. Opher, Phys. Rev. D63, 123508 (2001).

[56] T. Banks, M. Dine and A.E. Nelson, JHEP 06, 014 (1999). 
[57] E. Elizalde, Nuovo Cim. 104B, 685 (1989).

[58] L. Parker and A. Raval, Phys. Rev. D62, 083503 (2000).

[59] A.A. Saharian and M.R. Setare, Phys. Lett. B552, 119 (2003).

[60] J. Haro and E. Elizalde, All time physical approach to the dynamical Casimir effect, hep-th/0607186.

[61] E. Elizalde, J. Phys. A27, L299 (1994).

[62] V. Sahni and S. Habib, Phys. Rev. Lett. 81, 1766 (1998); L. Parker and A. Raval, Phys. Rev. D60, 063512 and 123502 (1999). 